\title{
Adherence to Guidelines: Experience of a Canadian Stroke Prevention Clinic
}

\author{
Abdullah Al-Salti, Lucy Vieira, Robert Côté
}

\begin{abstract}
Background: Few studies have assessed the performance of stroke prevention clinics. In particular, limited information exists on patient compliance, achievement of therapeutic targets, and related occurrence of vascular events. Methods: We compared our clinical practice to recommendations from published guidelines in newly referred patients for transient ischemic attack (TIA) or ischemic stroke between 2008 and 2010. We monitored our cohort for at least 1 year and assessed for adequacy of vascular risk factor management, drug adherence, and occurrence of nonlethal vascular outcomes. Results: Of 408 patients, $57.8 \%$ had a stroke and $42.2 \%$ a TIA. The mean age was $68 \pm 13$ years, and 52\% male. Average follow-up was 15.8 months. During follow-up, 253 patients (70.3\%) completely achieved their blood pressure target, $151(45.5 \%)$ achieved their low-density lipoprotein (LDL) cholesterol target, and 407 (99.8\%) were on antithrombotics. Eighty-nine patients $(21.8 \%)$ attained optimal therapy status, defined as reaching targets for LDL cholesterol, blood pressure, and antithrombotic use. Adherence to drug therapy was associated with attainment of optimal therapy status $(p=0.01)$. Diabetes was associated with lower probability of attaining optimal therapy status (odds ratio [OR], 0.36; 95\% confidence interval [CI], 0.20-0.66) and blood pressure targets (OR, 0.09; 95\% CI, 0.05-0.17). During follow-up, 52 (12.7\%) patients had a nonlethal vascular event. Conclusion: Our study shows good attainment of therapeutic goals associated with adherence to drug therapy. However, optimal therapy status and blood pressure targets were more difficult to attain in patients with diabetes; therefore, more intensive preventive efforts may be required for these individuals.
\end{abstract}

RÉSUMÉ: Adhésion aux lignes directrices: expérience d'une clinique canadienne de prévention de l'accident vasculaire cérébral. Contexte: Peu d'études ont évalué la performance des cliniques de prévention AVC. En particulier il existe peu d'information sur l'adhérence des patients, l'atteinte des cibles thérapeutiques et la survenue d'événements vasculaires. Méthode: Nous avons comparé notre pratique clinique aux lignes directrices publiées chez les nouveaux patients reférés pour attaque ischémique transitoire (AIT) ou AVC ischémique entre 2008 et 2010. Nous avons suivis notre cohorte sur au moins une année et évalué le contrôle des facteurs de risque vasculaires,adhérence aux médicaments et la survenue d'événements vasculaires non-mortels. Résultats: Chez 408 patients, $57.8 \%$ ont présenté un AVC et $42.2 \%$ un AIT. L'âge moyen était $68+/-13$ ans et $52 \%$ étaient des hommes. Le suivi moyen fut de 15.8 mois.Durant le suivi, 253 patients (70.3\%) ont atteints complètement leur cible de tension artérielle,151 (45.5\%) leur cible de lipoprotéine de basse-densité (LDL) du cholestérol et 407 (99.8\%) prenaient un antithrombotique. Quatre vingt neuf patients (21.8\%) ont atteints le statut de traitement optimal,défini comme atteinte des cibles pour le LDL cholestérol,tension artérielle et prise d'antithrombotiques. L'adhérence aux médicaments était associée à l'atteinte du statut optimal $(\mathrm{p}=0.01)$. Le diabète était associé avec une probabilité diminuée d'atteindre le statut de traitement optimal (OR,0.36;95\% interval de confiance (IC), 0.20-0.66) et d'atteinte de la cible de tension artérielle (OR, 0.09;95\% IC, 0.05-0.17). Durant le suivi, $52(12.7 \%)$ patients ont présenté un événement vasculaire non-mortel. Conclusion: Notre étude démontre une bonne atteinte des cibles thérapeutiques associée à l'adhérence médicamenteuse. Par ailleurs,l'atteinte du statut optimal et des cibles de tension artérielle étaient plus difficiles à atteindre chez les diabétiques; donc plus d'efforts préventifs seraient requis chez ces individus.

Keywords: Vascular risk factor, stroke prevention, drug adherence

doi:10.1017/cjn.2014.20

Can J Neurol Sci. 2014; 41: 562-567

\section{INTRODUCTION}

Stroke remains a major health care problem worldwide. In Canada, approximately 50,000 new cases of stroke are diagnosed annually and more than 300,000 Canadians are stroke survivors. ${ }^{1}$ In the United States, approximately 795,000 persons have a stroke each year, of which an estimated 180,000 are recurrent. ${ }^{2}$ Reducing the rate of recurrent stroke requires identifying and controlling modifiable risk factors in the early poststroke/transient ischemic attack (TIA) period and developing appropriate preventive strategies. ${ }^{1,3-6}$

The identification and control of modifiable vascular risk factors (MRFs) is one of the main goals of stroke prevention clinics (SPCs) in addition to appropriate use of antithrombotics and selective surgical interventions as suggested by current practice guidelines. ${ }^{1,6,7}$
Unfortunately, a relatively high proportion of patients fail to achieve the recommended therapeutic objectives. ${ }^{8-10}$ Recent evidence in the literature suggests that improving outpatient care through referral to SPCs may decrease risk of adverse outcomes. ${ }^{3}$

We retrospectively assessed the effectiveness and impact of our SPC in achieving therapeutic targets for vascular risk factors and appropriate medical and surgical interventions. We also

\footnotetext{
From the McGill University (AA-S, LV, RC), Montreal, Quebec, Canada.

Received February 11, 2014. Final Revisions Submitted April 11, 2014 Correspondence to: Robert Côté, Department of Neurology, McGill University, Montreal General Hospital, Rm. L7-408, Montreal, Quebec H3G 1A4, Canada. Email: robert.cote@mcgill.ca.
} 
assessed adherence to prescribed drug therapy and its impact on the attainment of therapeutic goals.

\section{METHODS}

To make comparisons, we used recommendations from the latest practice guidelines, ${ }^{1,5}$ from which we derived therapeutic targets of interest:

- Blood pressure (BP) (nondiabetic, <140/90 mmHg; diabetic, $<130 / 80 \mathrm{mmHg}$ )

- Lipids (low-density lipoprotein [LDL] cholesterol $<2.0 \mathrm{mmol} / \mathrm{L}$ )

- Appropriate use of antithrombotic therapy

- Cessation of smoking

We also recorded the proportion of patients with carotid interventions and their timing. We also assessed adherence to drug therapy and its association with attainment of risk factor control during follow-up.

\section{Patient Selection}

We included all recently symptomatic ( $<6$ months) patients consecutively referred for a TIA or nondisabling ischemic stroke between 2008 and 2010. Patients were referred from one of two affiliated teaching hospitals (Montreal General Hospital and Montreal Neurological Hospital) or from the community.

\section{Study Design}

This is an observational retrospective study. Relevant clinical information (except mortality) was collected for at least 1 year from the first assessment in the clinic. Sources of information included clinic charts, computerized hospital records, and clinical information from primary health care providers (family physicians). The information included demographic features, vascular risk factors, medication, and interventions related to the cerebrovascular condition. All information regarding new nonlethal vascular events was collected. All patient data were kept confidential and all analyses and reports are presented in aggregate form. This study was approved by the local research board of the McGill University Health Center research institute.

\section{Exclusion Criteria}

Patients were excluded from the study if they had a disabling stroke that limited follow-up, hemorrhagic stroke, remote stroke or TIA ( $>6$ months), or strokes associated with nonatherosclerotic causes (cerebral venous thrombosis, connective-tissue disease, complication from a systemic disease, arterial dissection, iatrogenic). Finally, patients with no documented vascular risk factors and undetermined cause (all diagnostic tests normal) for their ischemic event were also excluded as well as patients with only one initial visit and no follow-up.

\section{Data Collection}

At the first visit, demographics, clinical features, and results of diagnostic tests were collected. Diagnostic investigations included cervical/intracranial duplex, 24-48 h Holter, echocardiogram, electrocardiogram, cerebral neuroimaging (CT/CT angiography, MRI/MR angiography), and lipid and diabetic profiles. Clinical features included vascular risk factors, medications, smoking status, and carotid interventions. Ischemic stroke subtypes were categorized using the TOAST (Trial of Org 10172 in Acute Stroke Treatment) criteria. ${ }^{11}$

All patients were offered regular follow-up at our clinic. At each follow-up visit, assessment of vascular risk factors was determined as well as any intervention to optimize their control. Information was collected directly from the patient, hospital computerized chart, home measurements, or family physician offices or other clinics. New vascular events were recorded and smoking status verified. Adherence to drug therapy and the reason for nonadherence were also recorded.

\section{Hypertension}

Diagnosis of hypertension was based on history and use of antihypertensive medication. We based BP targets were based on recommendations from best-practice stroke guidelines. ${ }^{1,5}$ For nondiabetics, target was $<140 / 90 \mathrm{mmHg}$; for diabetics, $<130 / 80 \mathrm{mmHg}$. We also collected information about types of antihypertensives used.

\section{DLP and LDL Cholesterol}

Diagnosis of dyslipidemia (DLP) was based on history and use of lipid-lowering medication. We assessed lipid control by determining the fasting lipid profile, using the LDL cholesterol values $<2.0 \mathrm{mmol} / \mathrm{L}$. We also recorded information about the types of lipid-lowering medications.

\section{Diabetes}

Diagnosis of diabetes was based on history or fasting blood glucose levels and hemoglobin A1c results (glycated hemoglobin A1c level $\leq 7.0$, fasting plasma glucose target of 4.0-7.0 mmol). Because no specific targets exist for stroke prevention, we performed no statistical analyses on diabetic control. We also obtained information about types of diabetic medications.

\section{Smoking}

Patients were classified as nonsmokers, smokers, or ex-smokers.

\section{Adherence to Therapy}

Adherence to prescribed medications was assessed from the patient directly clinic chart, prescription sheets from pharmacies, and computerized hospital chart. Information regarding nonadherence was recorded including reasons for non-adherence.

\section{Carotid Intervention}

Patients who submitted to carotid interventions (surgery or stenting) were identified as well as the timing of the intervention.

\section{Outcomes}

Occurrence of vascular events, including TIA, all strokes, and coronary events, was recorded during follow-up. This information was collected directly from the patient and computerized hospital chart, which included visits to emergency departments or admissions to our hospitals or other institutions. Unfortunately, we could not reliably determine the occurrence of death because mortality information was not consistently available and we did not have access to provincial mortality databases. 


\section{Statistical Analysis}

For each parameter (LDL-C, BP, antithrombotic use), results were categorized in 3 groups:

1. Completely attained if at all follow-up visits and/or at the last documented follow-up visit (1 year or more) the target was attained for this parameter.

2. Partly attained if the target was attained only at some of the follow-up visits but not consistently.

3. Not attained if the target had never been attained at any time during follow-up.

Optimal therapy was defined as attainment of the targets for the three parameters (LDL-C, BP, antithrombotic use) as a group. Optimal therapy was completely attained if all three parameters were completely attained as defined above. Optimal therapy was partly attained if only one or two parameters were fully achieved or any parameter was only partly achieved. Optimal therapy was not attained if none of the parameters were attained at any time during follow-up.

Given a definition of subgroups, categorical variables (e.g., sex, hypertension, diabetes mellitus, DLP, coronary artery disease, smoking, adherence) were tabulated by frequency, by groups (completely attained vs. partially attained vs. not attained), and for all subjects. Continuous variables (e.g., age, BP, LDL cholesterol) were presented using summary statistics (mean, standard deviation) by groups and for all subjects. Fisher's exact test (for categorical variables) or Wilcoxon rank sum test (for continuous variables) was performed to assess homogeneity of baseline characteristics among the groups. All comparisons were carried out using a two-sided test at an $\alpha$ level of 0.05 unless otherwise specified. No formal adjustments were made for the multiplicity of inferences for multiple clinical/demographical features.

In addition, we used a proportional-odds model using prespecified factors (sex, age, adherence) as covariates to verify the impact of these factors on patient attainment of therapeutic goals for BP, LDL cholesterol, and optimal therapy and/or identify factors significantly related to the attainment of these parameters. For each parameter, a final model was determined using a stepwise model-building procedure with a significance level of 0.2 used for entry or removal of variables. Regression analyses excluded all subjects with missing values in any covariate. Statistical analyses including descriptive statistics and proportionalodds regressions were performed with SAS (version 9.2, SAS, Cary, NC, USA).

\section{Results}

Of the 800 new patients seen between January 2008 and December 2010, 392 were excluded for the following reasons: nonatherosclerotic etiology, 82 (21\%); age below 45 years, $8(2 \%)$; hemorrhagic stroke, $55(14 \%)$; remote stroke or TIA, 37 (9\%); disabling stroke, 2 (1\%); absence of follow-up visit, 188 (48\%); and undetermined cause of stroke without MRFs, 15 (4\%). Thus, our study included 408 patients: 236 strokes (57.8\%) and 172 TIAs (42.2\%), with an average follow-up of 15.8 months. The average number of visits per patient was 3 . The mean age was $68 \pm 13$ years, and $52 \%$ were male. Table 1 gives more demographics and clinical features.

A total of 47 patients $(11.5 \%)$ had a significant carotid stenosis $(\geq 70 \%)$, and of these $22(46.8 \%)$ had a carotid intervention
Table 1: Baseline characteristics

\begin{tabular}{|c|c|}
\hline Characteristics & Patients $(N=408),(\%)$ \\
\hline \multicolumn{2}{|l|}{ Sex } \\
\hline Female & $194(48 \%)$ \\
\hline Male & $214(52 \%)$ \\
\hline \multicolumn{2}{|l|}{ Age } \\
\hline Mean & $68 \pm 13$ \\
\hline \multicolumn{2}{|l|}{ Hypertension } \\
\hline No & $123(30.1 \%)$ \\
\hline Yes & $285(69.9 \%)$ \\
\hline \multicolumn{2}{|l|}{ Diabetes } \\
\hline No & $311(76.2 \%)$ \\
\hline Yes & $97(23.8 \%)$ \\
\hline \multicolumn{2}{|l|}{ Dyslipidemia } \\
\hline No & $171(41.9 \%)$ \\
\hline Yes & $237(58.1 \%)$ \\
\hline \multicolumn{2}{|c|}{ Coronary artery disease } \\
\hline No & $336(82.4 \%)$ \\
\hline Yes & $72(19.6 \%)$ \\
\hline \multicolumn{2}{|l|}{ Smoking } \\
\hline No & $339(83.1 \%)$ \\
\hline Yes & $69(16.9 \%)$ \\
\hline \multicolumn{2}{|l|}{ Atrial fibrillation } \\
\hline No & $355(87.0 \%)$ \\
\hline Yes & $53(13.0 \%)$ \\
\hline \multicolumn{2}{|l|}{ Stroke subtypes } \\
\hline Large artery & $107(26.2 \%)$ \\
\hline Cardioembolic & $60(14.7 \%)$ \\
\hline Small artery & $133(32.6 \%)$ \\
\hline Undetermined & $108(26.5 \%)$ \\
\hline \multicolumn{2}{|l|}{ No. Of MRF* } \\
\hline 0 & $58(14.2 \%)$ \\
\hline $1-2$ & $265(65.0 \%)$ \\
\hline$\geq 3$ & $85(20.8 \%)$ \\
\hline
\end{tabular}

*MRF: Modifiable risk factors

(carotid endarterectomy, $n=21$ and stenting, $n=1$ ) for symptomatic disease. Median time from the ischemic event to intervention was 25 days and only four patients $(18 \%)$ had the intervention performed within 14 days. Two hundred seventy-nine $(68.4 \%)$ patients were on antihypertensive medication, 295 (72.3\%) on a cholesterol-lowering agent, $94(23.0 \%)$ on a diabetic medication, and $407(98.5 \%)$ on an antithrombotic medication. Most patients had brain imaging, either CT $(98 \%)$ or MRI $(41 \%)$ or both, and most $(79 \%)$ had an echocardiogram and $91 \%$ a cervical/intracranial duplex; see Table 2. Overall during follow-up, 253 patients (70.3\%) completely attained their BP target, 151 (45.5\%) completely attained their LDL cholesterol target, and 407 (99.8\%) were on an antithrombotic medication. Eighty-nine patients $(21.8 \%)$ completely attained the optimal therapy status; see Table 3. 
Table 2: Investigations and drug therapy

\begin{tabular}{l|c|c}
\hline Drug therapy/investigations & Patients $(\boldsymbol{N}=\mathbf{4 0 8})$ & $\boldsymbol{\%}$ \\
\hline Antihypertensive & 279 & 68.4 \\
\hline Antithrombotic & 407 & 99.8 \\
\hline Lipid lowering & 295 & 72.3 \\
\hline Statin & 287 & 70.3 \\
\hline Diabetic & 94 & 23.0 \\
\hline Adherence to therapy & 390 & 95.6 \\
\hline CT brain & 400 & 98 \\
\hline CTA & 51 & 12.5 \\
\hline MRI & 167 & 41 \\
\hline MRA & 74 & 18 \\
\hline Duplex & 370 & 91 \\
\hline Echocardiogram & 323 & 79 \\
\hline Holter & 160 & 39 \\
\hline
\end{tabular}

CTA, CT angiography; MRA, MR angiography.

\section{Hypertension}

Overall, 285 patients (69.9\%) were hypertensive, and of these 279 $(97.9 \%)$ were on treatment. Angiotensin-converting enzyme inhibitors, diuretics and beta blockers, in that order, were the most common single or combined antihypertensive medications used. The overall mean BP during the follow-up period was $132 \pm 18 / 75 \pm 10 \mathrm{mmHg}$. Only $48(11.8 \%)$ patients were missing BP recordings. Analysis of the remaining $360(88.2 \%)$ patients showed that $253(70.3 \%)$ completely attained BP target, $28(7.8 \%)$ had only partial attainment, and $79(21.9 \%)$ did not attain BP target. Mean BP level for the completely attained group was $128 \pm 16 / 73 \pm 10 \mathrm{mmHg}$, $134 \pm 15 / 76 \pm 9 \mathrm{mmHg}$ for the partially attained group, and $145 \pm 19 / 79 \pm 9 \mathrm{mmHg}$ for the nonattained group.

\section{DLP and LDL Cholesterol}

A total of 237 patients $(58.1 \%)$ had a diagnosis of DLP at baseline, and $295(72.3 \%)$ patients were treated with a lipidlowering agent. Overall, most treated patients $(97.3 \%)$ were on statin therapy. Seventy-six patients (18.6\%) had missing LDL cholesterol values. The overall mean LDL cholesterol level was $2.8 \pm 1.1 \mathrm{mmol} / \mathrm{L}$. Of the 332 patients with available values, 151 patients $(45.5 \%)$ completely attained their LDL target, $7(2.1 \%)$ only partially attained it, and $174(52.4 \%)$ did not attain it.

Table 3: Attainment of therapeutic targets

\begin{tabular}{l|c|c|c}
\hline Parameter & CA & PA & NA \\
\hline BP target, $n(\%)$ & $253(70.3)$ & $28(7.8)$ & $79(21.9)$ \\
\hline LDL cholesterol, $n(\%)$ & $151(45.5)$ & $7(2.1)$ & $174(52.4)$ \\
\hline Antithrombotic use, $n(\%)$ & $407(99.8)$ & $0(0)$ & $1(0.2)$ \\
\hline Cessation of smoking, $n(\%)$ & $50(72.5)$ & - & $19(27.5)$ \\
\hline Optimal therapy, $n(\%)$ & $89(21.8)$ & $319(78.2)$ & $0(0)$ \\
\hline
\end{tabular}

CA, completely attained; PA, partially attained; NA, not attained; BP, blood pressure; LDL, low-density lipoprotein. - , no data.
The median LDL cholesterol level in the completely attained group was $1.9 \mathrm{mmol} / \mathrm{L}, 2.8 \mathrm{mmol} / \mathrm{L}$ in the partially attained group, and $3.3 \mathrm{mmol} / \mathrm{L}$ in the nonattained group.

\section{Optimal Therapy}

Eighty-nine patients $(21.8 \%)$ completely attained the status of optimal therapy, and $316(78.2 \%)$ were classified as partially attained. No patient was classified as not attained because virtually all patients were on an antithrombotic medication.

Univariate analysis showed that sex and age had no significant association with attainment of optimal therapy as well as number of MRFs and stroke subtypes. However, adherence to prescribed drug therapy showed a significant association with attainment of optimal therapy status $(p=0.043)$.

\section{Smoking}

Fifty of $69(72.5 \%)$ of patients stopped smoking. However, smoking cessation showed no significant association with attaining either BP targets or optimal therapy status $(p=0.397$ and 0.860 , respectively). However, smokers were less likely to attain their LDL cholesterol target than were nonsmokers $(p=0.009)$.

\section{Adherence to Therapy}

Overall, 390 patients $(95.6 \%)$ adhered to medical therapy. Only 18 patients $(4.4 \%)$ were nonadherent to one or multiple medications. Side effects of drugs (mostly statins) were the main reasons for decreased adherence.

\section{Multivariate Analysis \\ BP Target Attainment}

The presence of hypertension or diabetes was associated with a lower probability of attaining BP targets (OR, 0.39 [95\% CI, 0.18-0.83] and OR, 0.09 [95\% CI, 0.05-0.17]), respectively. However, DLP was associated with a higher probability of attaining BP targets: OR, 2.33 (95\% CI, 1.25-4.35).

\section{LDL Cholesterol Target Attainment}

Diabetes or DLP was associated with a higher probability of attaining the LDL cholesterol target (OR, 2.10 [95\% CI, 1.12-3.94] and OR, 2.10 [95\% CI, 1.24-3.56]), respectively, as well as adherence to drug therapy (OR, 4.9 [95\% CI, 0.95-25]). A higher LDL cholesterol value at baseline and smoking were both associated with a lower probability of achieving the LDL targets (OR, 0.42 [95\% CI, 0.320-0.554] and OR, 0.33 [95\% CI, $0.16-0.67])$, respectively.

\section{Optimal Therapy Status Attainment}

Diabetes was associated with a lower probability of attaining optimal therapy status (OR, 0.36 [95\% CI, 0.20-0.66]) as well as a higher systolic BP and LDL cholesterol level at baseline (OR, 0.98 [95\% CI, 0.97-1.0]) and OR, 0.58 [95\% CI, 0.45-0.74]), respectively. However, DLP and adherence to drug therapy were both associated with a higher probability of attaining optimal therapy (OR, 2.34 [95\% CI, 1.37-4.0] and OR, 6.58 [95\% CI, 1.56-27.78]), respectively (Table 4). 
Table 4: Multivariate analysis of factors associated with attainment of optimal therapy

\begin{tabular}{l|c|r}
\hline Factor & OR $(\mathbf{9 5 \%}$ CI) & $\boldsymbol{p}$ \\
\hline Diabetes & $0.36(0.20-0.66)$ & 0.001 \\
\hline DLP & $2.34(1.37-4.0)$ & 0.002 \\
\hline SBP & $0.98(0.97-1.0)$ & 0.009 \\
\hline LDL & $0.58(0.45-0.74)$ & $<0.001$ \\
\hline Adherence to therapy & $6.58(1.56-27.78)$ & 0.010 \\
\hline
\end{tabular}

CI, confidence interval; DLP, dyslipidemia; SBP, systolic blood pressure; LDL, low-density lipoprotein.

\section{Outcomes}

During follow-up, 52 patients (12.7\%) presented with a new vascular event. We recorded 59 vascular events (seven patients had more than one event). This group included 17 patients (32.7\%) with a stroke, (ischemic or hemorrhagic), 14 patients (26.9\%) with a TIA, and $14(26.9 \%)$ with a coronary event. Three patients $(5.8 \%)$ had both a TIA and a stroke, and four (7.7\%) had both a TIA and a coronary event.

The vascular event (TIA, all strokes, and coronary events)free survival curves are presented in Figure 1, comparing the completely attained group to the not-completely attained group for optimal therapy status. The difference between the two groups was not significant $(p=0.589)$.

\section{Discussion}

Our study shows that most patients available for follow-up in our prevention clinic were adequately managed in regard to control of vascular risk factors, antithrombotic medications, and required carotid interventions. Also, most patients completed appropriate basic investigations.

Overall, more than $70 \%$ of patients completely attained their BP target and almost half completely attained their LDL cholesterol target. As well, virtually all patients were on an antithrombotic medication during follow-up. In the multivariate analysis, however, patients with diabetes showed a lower

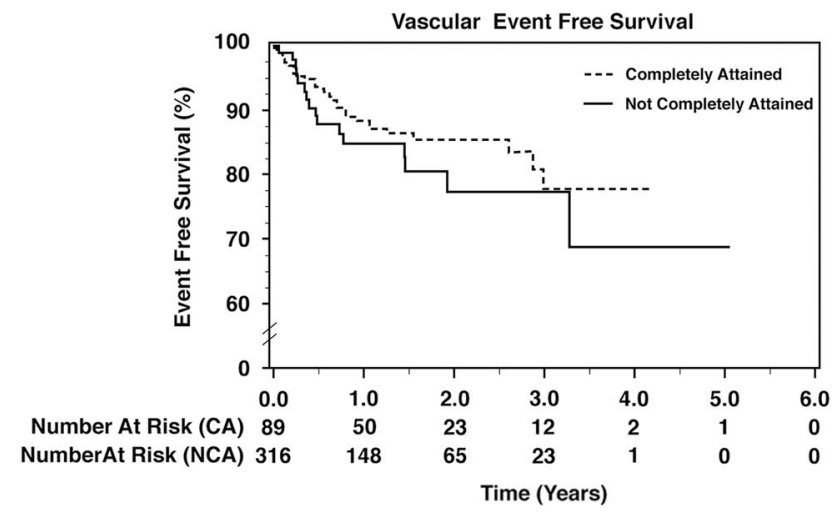

Figure 1: Event-free survival for patients who completely attained therapeutic target (CA) versus those who did not completely attain target (NCA). Comparison between groups did not show a significant difference $(p=0.589)$. probability of attaining their BP target and attaining the optimal therapy status. This could have been in part due to a more stringent therapeutic goal for BP control.

We also found very good adherence to drug therapy globally, and this was associated with a greater likelihood of reaching the optimal therapy status $(p=0.01)$. Unfortunately, one in four smokers (27.5\%) continued to smoke during the follow-up period, suggesting that more efforts are needed toward smoking-cessation strategies.

In a multicenter, prospective, cohort study of ambulatory patients with established cardiovascular disease, $45.3 \%$ of patients reached the recommended values for BP control. ${ }^{12}$ Compare this value with the more than $70 \%$ in our study; we also found that more than $45 \%$ of our patients completely achieved the recommended LDL cholesterol target, compared with $19.4 \%$ in that multicenter study. This outcome occurred even though the LDL cholesterol target was higher in the multicenter study $(\leq 2.5 \mathrm{mmol} / \mathrm{L})$ than in our study $(<2.0 \mathrm{mmol} / \mathrm{L})$. Both studies had similar proportions of patients on antithrombotic medication $(>90 \%)$. The proportion of patients who completely attained the optimal therapy status was $17.7 \%$ in the multicenter study, compared with $21.8 \%$ in our study.

In a small retrospective study based in a stroke clinic of a tertiary medical center, $51 \%$ of patients achieved the desired BP target and $45 \%$ achieved the LDL cholesterol target $(<3.4 \mathrm{mmol} / \mathrm{L}){ }^{13}$ However, the neurologists in the clinic relied on the primary-care physicians to prescribe therapy. In a large audit, in the UK, ${ }^{14} 41 \%$ of stroke patients achieved their BP target and $61 \%$ were still smoking. In another study looking at the cessation of smoking after a first stroke, only $21.7 \%$ of smokers gave up smoking within 6 months after their stroke, ${ }^{15}$ compared with more than $70 \%$ in our study.

For vascular outcomes, our study showed a relatively lower risk of events than that in previous studies despite a longer followup period. ${ }^{13}$ This finding could be explained by both the reported decline in vascular risk in recent years, possibly related to improved vascular preventive measures, ${ }^{16,17}$ and limited access to mortality data in our study.

The event-free survival rates for our study showed no significant difference between groups that completely attained optimal therapy status and those who did not $(p=0.589)$. This could have been due to a relative lack of statistical power related to a relatively lower number of events.

Finally, our wait times for carotid surgery, although better than recent reports, ${ }^{18}$ still were not within the recommended 2-week period.

\section{Strengths and Limitations}

Strengths of our study include the relatively large number of individuals, length of follow-up, and the good quality of clinical information. However, limitations also exist. First, this was a retrospective and observational study, making it more liable to biases. Second, we did not have full access to mortality data, thereby affecting the number of outcome events reported. Third, owing to the design of our study, conclusions are limited to patients who attended regular follow-ups and cannot apply to all ambulatory patients after a TIA or nondisabling ischemic stroke. Finally, apart from smoking, we did not collect any specific information on other potentially important lifestyle factors such as physical activity or diet. 


\section{CONCLuSION}

Our study showed good overall attainment of different therapeutic targets in a large proportion of patients when compared to best-practice recommendations. ${ }^{1,5}$ Overall, adherence to prescribed medication was very good and associated with attainment of optimal therapy status. These results support the effectiveness of a specialized SPC in managing this high-risk population, although we could not show a direct effect on vascular outcomes. Also, diabetic patients were less likely to attain their therapeutic targets, so more emphasis on preventive measures is required in these individuals.

\section{ACKNOWLEDGMENTS}

We thank Sandy Lavigne, secretary at SPC MUHC, and Lisa Wadup, stroke nurse at McGill General Hospital.

\section{Disclosures}

The authors report no disclosures.

\section{REFERENCES}

1. Lindsay MP, Gubitz G, Bayley M, Phillips S, editors on behalf of the Canadian Stroke Best Practices and Standards Working Group. Canadian best practice recommendations for stroke care. Canadian Stroke Network; 2012 [cited 2013 Nov 21]. Available from http://www.strokebestpractices.ca.

2. Lloyd-Jones D, Adams RJ, Brown TM, et al. Heart disease and stroke statistics-2010 update: a report from the American Heart Association. Circulation. 2010;121:e46-215.

3. Webster F, Saposnik G, Kapral MK, Fang J, O'Callaghan C, Hachinski V. Organized outpatient care: stroke prevention clinic referrals are associated with reduced mortality after transient ischemic attack and ischemic stroke. Stroke. 2011;42:3176-82.

4. Rahiman A, Saver JL, Porter V, et al. In-hospital initiation of secondary prevention is associated with improved vascular outcomes at 3 months. J Stroke Cerebrovasc Dis. 2008;17:5-8.

5. Furie KL, Kasner SE, Adams RJ, et al. Guidelines for the prevention of stroke in patients with stroke or transient ischemic attack: a guideline for healthcare professionals from the American Heart Association/American Stroke Association. Stroke. 2011; 42:227-76.
6. Mouradian MS, Majumdar SR, Senthilselvan A, Khan K, Shuaib A. How well are hypertension, hyperlipidemia, diabetes, and smoking managed after a stroke or transient ischemic attack? Stroke. 2002;33:1656-9.

7. Touzé E, Coste J, Voicu M, et al. Importance of in-hospital initiation of therapies and therapeutic inertia in secondary stroke prevention implementation of prevention after a cerebrovascular event (IMPACT) Study. Stroke. 2008;39:1834-43.

8. Bushnell CD, Zimmer LO, Pan W, et al. Persistence with stroke prevention medications 3 months after hospitalization. Arch Neurol. 2010;67:1456-63.

9. Wei JW, Wang JG, Huang Y, et al. Secondary prevention of ischemic stroke in urban China. Stroke. 2010;41:967-74.

10. Ovbiagele B, Saver JL, Fredieu A, et al. In-hospital initiation of secondary stroke prevention therapies yields high rates of adherence at follow-up. Stroke. 2004;35:2879-83.

11. Kolominsky-Rabas PL, Weber M, Gefeller O, Neundoerfer B, Heuschmann PU. Epidemiology of ischemic stroke subtypes according to TOAST criteria: incidence, recurrence, and long-term survival in ischemic stroke subtypes: A populationbased study. Stroke. 2001;32:2735-40.

12. Saposnik G, Goodman SG, Leiter LA, et al. Applying the evidence: do patients with stroke, coronary artery disease, or both achieve similar treatment goals? Stroke. 2009;40:1417-24.

13. Joseph LN, Babikian VL, Allen NC, Winter MR. Risk factor modification in stroke prevention. The experience of a stroke clinic. Stroke. 1999;30:16-20.

14. Rudd AG, Lowe D, Hoffman A, Irwin P, Pearson M. Secondary prevention for stroke in the United Kingdom: results from the National Sentinel Audit of Stroke. Age Ageing. 2004;33: 280-6.

15. Bak S, Sindrup SH, Alslev T, Kristensen O, Christensen K, Gaist D. Cessation of smoking after first-ever stroke. A follow-up study. Stroke. 2002;33:2263-9.

16. Marquardt L, Geraghty OC, Mehta Z, Rothwell PM. Low risk of ipsilateral stroke in patients with asymptomatic carotid stenosis on best medical treatment: a prospective, population-based study. Stroke. 2010;41:e11-e17.

17. Goessens BMB, Visseren FLJ, Kappelle LJ, Algra A, van der Graaf Y. Asymptomatic carotid artery stenosis and the risk of new vascular events in patients with manifest arterial disease: the SMART study. Stroke. 2007;38:1470-5.

18. Jetty P, Husereau D, Kubelik D, et al. Wait times among patients with symptomatic carotid artery stenosis requiring carotid endarterectomy for stroke prevention. J Vasc Surg. 2012;56:661-7. 